Mini Review

\section{Progress of chemical components and biological activities of Fructus}

\section{Amomi}

\section{Yuchen Xiao ${ }^{1,3,4}$, Olagoke Zacchaeus Olatunde ${ }^{1,4}$, Jianping Yong $^{2 *}$ and Canzhong Lu' ${ }^{1-3 *}$}

\author{
${ }^{1}$ Fujian Institute of Research on the Structure of Matter, Haixi Institute, Chinese Academy of \\ Sciences, China \\ ${ }^{2}$ Xiamen Institute of Rare-earth Materials, Haixi Institute, Chinese Academy of Sciences, China \\ ${ }^{3}$ Shanghai Tech University, China \\ ${ }^{4}$ University of Chinese Academy of Sciences, China
}

\section{Abstract}

Fructus Amomi is "one of the top four south authentic Traditional Chinese Plant Medicines", widely distributed in tropical and subtropical districts of China, such as Fujian, Guangdong, Guangxi, Hainan, etc., which has ever been used in clinical for treatment of the digestive diseases. In addition, it has also been used as spices. In recent years, some research about its components together with pharmacological activities have been reported. In this Mini review, we mainly describe the recent progress of the components and pharmacological activities of Fructus Amomi, to provide the reference for the relevant researchers.

\section{More Information}

*Address for Correspondence: Canzhong Lu, Fujian Institute of Research on the Structure of Matter, Xiamen Institute of Rare-earth Materials, Haixi Institute, Chinese Academy of Sciences, China, Tel: +86-592-3576166;

Email: czlu@fjirsm.ac.cn

Jianping Yong, Xiamen Institute of Rare-earth Materials, Haixi Institute, Chinese Academy of Sciences, China, Tel: +86-591-63173162; Email: jpyong@fjirsm.ac.cn

Submitted: 05 March 2020

Approved: 12 March 2020

Published: 13 March 2020

How to cite this article: Xiao $\mathrm{Y}$, Olatunde $\mathrm{OZ}$, Yong J, Lu C. Progress of chemical components and biological activities of Fructus Amomi. Arch Biotechnol Biomed. 2020; 4: 001-004.

DOI: 10.29328/journal.abb.1001015

Copyright: (c) 2020 Xiao Y, et al. This is an open access article distributed under the Creative Commons Attribution License, which permits unrestricted use, distribution, and reproduction in any medium, provided the original work is properly cited.

Keywords: Fructus Amomi; Chemica components; Pharmacological activities

(D) Check for updates

OPEN ACCESS

\section{Introduction}

Fructus Amomi is "one of the top four south authentic Traditional Chinese Plant Medicines". It is also an important species included in Chinese Pharmacopoeia (2010). Fructus Amomi is a perennial herb of amomum, zingiberacease, which has a history of over 1300 years in medicine and food [1]. It is widely distributed in tropical and subtropical districts of China, such as Fujian, Guangdong, Guangxi, Hainan, Yunnan etc [2] and southeast Aisa. However, there are some differences about the names of the Fructus Amomi planted in different places: Amomun villosum Lour. (Planted in Guangdong province, China), A. villosum Lour. var, xanthioides T. $L . W u$ et Senjen (Planted in Yunnan province, China; some also planted in southeast Aisan), A. longiligulare T. L. Wu (Planted in Hainan province, China) and A. xanthioides Wall (Planted in southeast Asian) [3]. Modern pharmacological studies showed that Fructus Amomi has ever been used in clinical for treatment of digestive diseases: such as protecting gastric mucusa, improving gastrointestinal function, relieving pain, preventing diarrhea, promoting the secretion of digestive juice and so on. It is reported that the quality and medical efficacy of Amomun villosum Lour planted in Guangdong province is with much higher activities than others $[4,5]$.

Some work about the chemical components together with pharmacological activities of Fructus Amomi have been reported. In this minireview, we focused on describing the progress of its chemical components and pharmacological activities to provide the reference for the relevant researchers.

\section{Chemical components}

The main chemical component of Fructus Amomi is volatile oil. Other chemical components such as flavonoids, carbohydrate, organic acids and inorganic components were also isolated and reported.

Volatile oil: Zhang, et al. [6] extracted the volatile oils from the dried seeds and fruit shells of Fructus Amomi respectively, and identified their chemical components and the contents by GC-MS. The results showed that 138 chemical constituents were identified from the four different volatile oils. The main components and their contents are: bornyl acetate (5\% - 47\%), camphor $(4 \%-17 \%)$, borneol $(1.5 \%-6 \%)$, camphene $(0.2 \%$ $3 \%)$, alpha-pinene $(0.2 \%-3 \%)$, beta-pinene $(0.2 \%-5 \%)$ and alpha-copaene $(0.1 \%-2 \%)$ (Representative structures listed in figure 1). The content of the total alkene accounts for $10 \%-40 \%$.

$\mathrm{Yu}$, et al. [7] obtained the fat-soluble fraction from the extraction of Amomun villosum Lour by silica gel 
chromatography, and identified its components by GC-MS. The results showed that the compounds of the contents over $1 \%$ are: bornyl acetate, camphor, camphor, cinene, camphorene, alpha-terpineol, myrcene and camphene (their structures listed in figure 2).

Zeng, et al. [8] obtained the volatile oils from the Amomun villosum Lour, A. villosum Lour. var. xanthioides T. L. Wu et Senjen and $A$. xanthioides Wall respectively, and identified their chemical components and the contents respectively. The results showed that bornay acetate is higher amount in volatile oil of Amomun villosum Lour. With the content of $59.6 \%$, while camphor is the highest amount in the volatile oil of A. villosum Lour. var. xanthioides T. L. Wu et Senjen and A. xanthioides Wall, with the contents of $63.02 \%$ and $60.23 \%$ respectively.

Flavonoids: Quercetin-3-rohamnoside, isoquercitrin and quercetin (Figure 3) were isolated and confirmed from the water-soluble extraction of Amomun villosum Lour. [9,10].

Carbohydrate: Fan, et al. [11] identified four monosaccharides (arabinose, mannose, glucose and galactose) (Figure 4) from Amomun villosum Lour and the relative proportion of these four monosaccharides was 1:0.68:0.97:0.81.

Organic acids: The main organic acids in Fructus Amomi are vanillic acid, stearic acid and palmitic acid (Figure 5) [7].

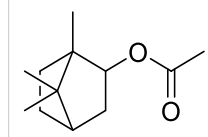

bornyl acetate

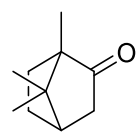

camphor

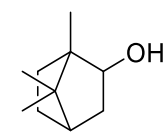

borneol

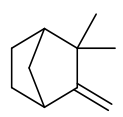

camphene

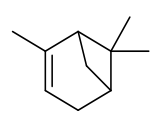

alpha-pinene

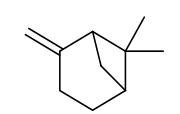

beta-pinene

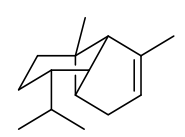

alpha-copaene
Figure 1: Representative chemical structures in volatile oil.

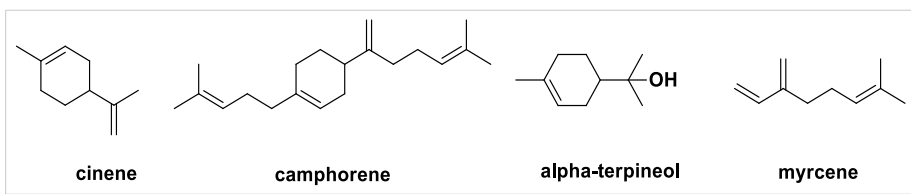

Figure 2: Chemical structures in fat-soluble components.

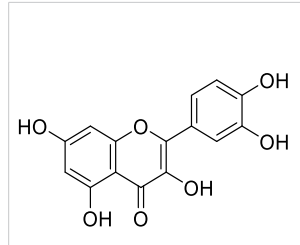

quercetin

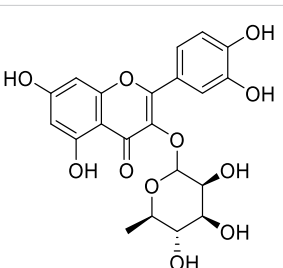

quercetin-3-rohamnoside

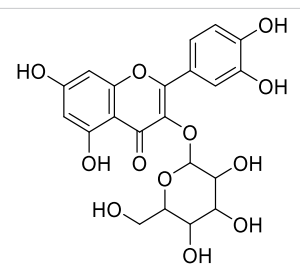

isoquercitrin
Figure 3: Flavonoids isolated from Amomun villosum Lour.
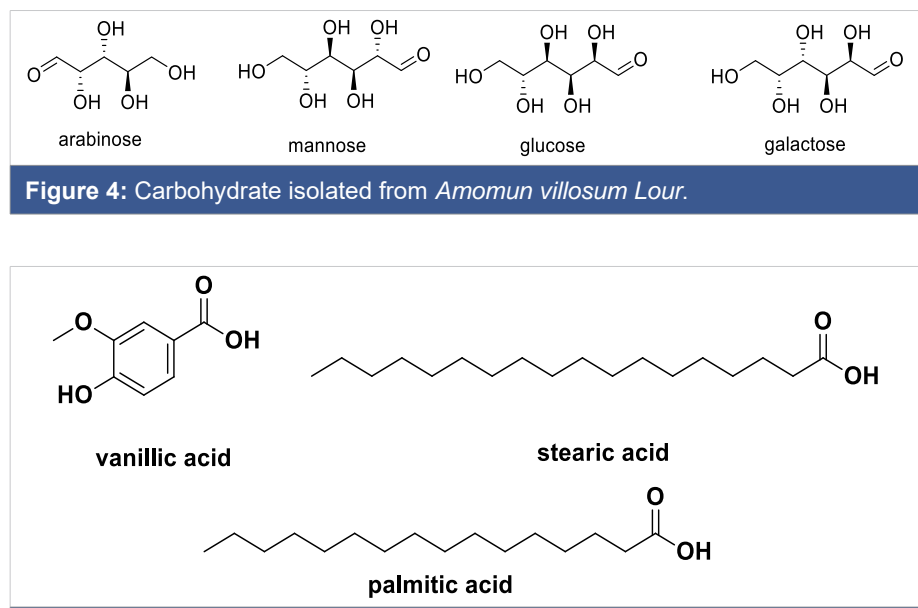

Figure 5: Ogranic acids isolated from Amomun villosum Lour.

Table 1: Correlation between the content of inorganic elements and volatile oil.

\begin{tabular}{|c|c|c|c|}
\hline \multirow{2}{*}{ Contents } & \multicolumn{3}{|c|}{ Amomun villosum Lour } \\
\hline & place $1^{\mathrm{a}}$ & place $2^{b}$ & place $3^{c}$ \\
\hline $\operatorname{Mn}\left(\times 10^{-6}\right)$ & 486.0 & 330.2 & 343.8 \\
\hline $\mathrm{Zn}\left(\times 10^{-6}\right)$ & 107.3 & 78.4 & 84.9 \\
\hline Volatile oil (\%) & 3.70 & 3.52 & 3.24 \\
\hline
\end{tabular}

Inorganic components: $\mathrm{Wu}$, et al. [12] determined the trace elements in Fructus Amomi and the trace elements are: cobalt, lead, nitrogen, silver, magnesium, potassium, iron, boron, copper, nickel, zinc, manganese and phosphorus. The elements content of zinc and manganese was positively correlated with the quality of the volatile oil (Table 1).

\section{Pharmacological activities}

Fructus Amomi is "one of the top four south authentic Traditional Chinese Plant Medicines". Different biological studies of Fructus Amomi have been carried out and it exhibited a wide spectrum of biological activities, such as: antibacteria, antiulcer effect, effect on gastrointestinal motility, effect of bioelectricity on gastrointestinal cells, sedative, anti-inflammatory, antidiarrheal effect, and anti-oxidation activities. The details list below.

Anti-bacteria activity: Zhang, et al. [6] obtained the volatile oil from the Fructus Amomi and tested its biological activities against fungi and bacteria. The results showed that the volatile oil exhibited significant inhibition to trichophyton rubrum, trichophyton mentagrophytes, microsporum gypseum, staphylococcus aureus and enterococcus faecalis. What's the most important is that the volatile oil expressed different inhibitory effects on different fungi and bacteria: the inhibitory efficacy on fungi is better than bacteria, while the efficacy on grampositive bacteria is better than gram-negative bacteria. Tang, et al. [14] found that some extractions of Fructus Amomi exhibited higher inhibition to klebsiella, staphylococcus, pseudomonas aeruginosa, salmonella, escherichia coli and bacillus subtilis. In addition, they also exhibited strong antioxidation activity. Yan, et al. [15] found that the water extraction of Fructus Amomi expressed significant restoring effect on the imbalance of intestinal flora caused by antibiotics. Cao, et al. [16] obtain 
the different extractions (petroleum ether extraction, ethyl acetate extraction, n-butanol extraction and water layer) and evaluated their antibacterial activity. The results showed that different extraction exhibited different antibacterial efficacy: the antibacterial efficacy sequences were: petroleum ether extraction $>$ ethyl acetate extraction $>n$-butanol extraction $>$ water layer. It means that the nonpolar extraction is more potent than the polar extractions.

Antiulcer effect: It is reported that some active components of the Fructus Amomi could inhibit gastric mucosal cell dysfunction and acute gastric mucosal injury (caused by hydrochloric acid) through inhibiting proteins and enzymes in gastric ulcers [17]. Gao, et al. [18] reported that the extraction of $A$. longiligulare T. $L$. $W u$ could protect the gastric mucosal injury of the rats and the mechanism may be related to improving the expression of TFF1 and TFF1 RNA proteins. Jafri, et al. [19] reported that the volatile oil of Fructus Amomi could reduce the secretion of gastric acid and pepsin, which had a protective effect on gastric mucosa.

Effect on gastrointestinal motility: Zhu, et al. [20] found that the extraction of Fructus Amomi could significantly promote the gastric emptying and intestinal transport, and its prokinetic efficacy was almost the same as the reference drug cisapride. Zhang, et al. [21] observed and recorded the effect of Fructus Amomi on 40 patients with functional dyspepsia. He found that Fructus Amomi could quickly alleviate the clinical symptoms of patients, while the main mechanism maybe that the Fructus Amomi could promote releasing the substance P and motilin.

Effect of bioelectricity on gastrointestinal cells: Fructus Amomi can affect the bioelectric activity of gastrointestinal cells. Ding, et al. [22] treated the rats (with syndrome of deficiency of spleen qi) with the water extraction of Fructus Amomi. The results showed that the water extraction of Fructus Amomi could increase the amplitude of pacemaker potential, thereby affecting the electrical activity of Cajal cells, repairing the damage of Cajal mesenchymal cells and signal pathway in small intestine of rats with syndrome of deficiency of spleen qi and improving gastrointestinal motility disorders.

Sedative, anti-inflammatory and antidiarrheal effect: Li, et al. [23] reported that bornyl acetate, the main component in the volatile oil of Fructus Amomi, could inhibit the diarrhea, relieved the pain of the tested mice and smooth muscle movement in isolated small intestine of the tested rabbits. But it did not show the significant effect on gastric emptation of the tested mice. $\mathrm{Wu}$, et al. [24] reported that the extraction of Fructus Amomi expressed significant analgesic and antiinflammatory activities. Zhang, et al. [25] evaluated the effects of the volatile oil from Fructus Amomi on intestinal mucositis induced by 5 -fluorouracil (5-FU). The results showed that Fructus Amomi and bornyl acetate significantly increased the rats' body weight, relieved diarrhea, and reversed histopathological changes in the gut and inflammation.
Ding, et al. [26] obtained the volatile oil of Amomun villosum Lour planted in Yunnan, and studied its antidiarrheal activity. The result showed that the volatile oil exhibited higher antidiarrheal activity in dose-dependent manner, and the highest dose is $270 \mathrm{mg} / \mathrm{kg}$. Lee, et al. [27] reported that the extraction of Fructus Amomi showed strong antiviral activity and protected cell survival in CVB3 infection.

Anti-oxidation: Sand kernel possess higher antioxidant activity and can be used as a safe and cheap natural antioxidant [28]. Zhao, et al. [29] reported that A. longiligulare T. L. Wu exhibited good antioxidant activity. Zhang, et al. [30] reported that the polysaccharides isolated from Fructus Amomi possessed a strong free radical scavenging activity, and it could significantly inhibit the formation of malondialdehyde in vitro and enhance antioxidant enzyme activity in mice with liver injury induced by carbon tetrachloride.

Other effects: Xiong, et al. [31] reported that the low dose of salt sunburn Fructus Amomi had the significant effect on reducing urine of the water-loaded mouse model, and preliminarily confirmed the accuracy of the processing theory of traditional Chinese medicine "salt sunburn into kidney" through pharmacological experiments. Huang, et al. [32] showed that the oil of Fructus Amomi leaf could significantly shorten the wound healing cycle of rabbits and promote wound healing with intact new epidermis, fewer inflammatory cells and less tissue lesions.

Zhao, et al. [33] reported that water extraction of $A$. villosum Lour. var. xanthioides T. L. Wu et Senjen could reduce the blood glucose of diabetic rats. Lee, et al. [34] reported that A. villosum $L$ could be used to treat the growth retardation during adolescence by the experiment (Amomum villosum induces longitudinal bone growth in adolescent female rats). It is also reported that Amomun villosum Lour exhibited higher inhibitory effects on transplanted tumors (S180 and H22) of the experimental mice during anti-tumor experiments, while it did not exhibit remarkable effect on the immune function of the mice [35].

\section{Conclusion}

Fructus Amomi is "one of the top four south authentic traditional Chinese medicines", widely used in clinical in China. Its main functions are to moisten the appetite, warm the spleen and prevent diarrhea, regulate qi and tocolysis. There are many kinds of components of Fructus Amomi together with a variety of pharmacological activities. In recent years, some chemical isolation and pharmacological studies of the Fructus Amomi have been carried out. But there are also some imperfections: (1) no new structures of compounds were isolated; (2) the action sites and the mechanism of pharmacological activities are not clear; (3) most of the work focused on the volatile oil, the research on other components and their biological activities are rare. Thus, we should carry out the deep work about the isolation of new structure compounds and further biological evaluation, to develop the 
new drugs or drug candidates from this Chinese Traditional Plant Medicines.

\section{Acknowledgment}

This work was financially supported by the National Natural Science Foundation of China (21875252) and Selfcreated Area Project of Major Science Technology Innovation Platform of Xiamen (3502ZCQ20171002).

\section{References}

1. Chinese Pharmacopoeia Commission. Chinese Pharmacopoeia. Chin Medical Sci Press. 2015; 253.

2. Chen J, Ding $P, X u X C$. Investigation of drug source and determination of Fructus Amomi. J Chinese Medicinal Materials. 2001; 24:18.

3. Lu SD, Wang J. The selling trend of Amomun villosum Lour is good year after year. Mordern Chin Med. 2008; 10: 48-49.

4. Yang CX. A comparative study on the chemical composition and main pharmacodynamics of Amomun villosum Lour from Yunnan and imported Fructus Amomi. Yunnan University of Traditional Chinese Medicine. 2017.

5. Fu C, Chen C, Zhou GX. Study on chemical constituents of Amomun villosum Lour. Chinese Traditional and Herbal Drugs. 2011; 42: 24102412.

6. Zhang ST, Wang ZY, Jiang TS. The chemical composition and antibacterial activity of the volatile oil of Fructus Amomi. Natural Product Research and Development. 2011; 23: 464-472.

7. Yu JG, Sun L, Zhou LD. Study on the chemical consituents of traditional Chinese medicine Fructus Amomi. Chin J Traditional Chin Med. 1997; 22: 231-232.

8. Zeng Z, Xi ZC, Meng SJ. Study on volatile components and quality evaluation of different varieties of Fructus Amomi. J Instrumental Analy. 2010; 29: 701-706.

9. Sun L, Yu JG, Zhou LD. Flavonoids glycoside compounds in traditional Chinese medicine Fructus Amomi. Chin J Chinese Materia Medica. 2002; 27: 40-42

10. Li ZZ, Pan RL, Li Z. The detection of total flavonoids, isoquercitrin and quercetin-3-rohamnoside in the Amomun villosum Lour. Science \& Technology Review. 2009; 27: 30-33.

11. Fan $\mathrm{YN}$, Huang $\mathrm{XL}$, Cheng $\mathrm{YH}$. Extraction and component analysis of polysaccharides from Amomun villosum Lour. J Guangzhou University. 2006; 5: 30-32.

12. Wu Z. Fuzzy clustering analysis of macroscopical and microelement characteristics of Fructus Amomi and its adulterants. J Chin Medicinal Materials. 2000; 23: 208

13. Huang $S Q$, Huang $H B$, Zeng $H$. Study on the inorganic components and pharmacodynamics of Amomun villosum Lour. Guangdong Trace Elements Science. 1994; 1: 27-34.

14. Tang JY, Liu FJ, Su MX. Study on the antibacterial and antioxidant effects of Fructus Amomi extract. Journal of Xiamen University. 2012; 51: 789-792.

15. Yan $Y$, Jin ML, Zhou L. Discussion on regulating effect of Fructus Amomi on intestinal flora imbalance mice caused by antibiotics. Chin J Microecol. 2013; 25: 1040-1043.

16. Cao GH, Zhang X, Wang XF. Comparison of antibacterial effect of different solvent extracts of Amomun villosum Lour and Amomun villosum Lour by salt processed. Science and Technology of Food Industry. 2019.
17. Huang GD, You N, Huang AH. Effect on volatile oil on gastrointestinal and VIP expression. J Chin Medicinal Materials. 2009; 32: 1587-1589.

18. Gao $Y$, Sun $T$, Xie $Y Q$. Effect of Amomunlongiligulare on TFF1 and TFF1 mRNA of gastric mucosa in rats with hep-atogeniculcer. Shanxi J Traditional Chin Med. 2016; 32: 52-54.

19. Jafri MA, Farah JK. Evaluation of the gas-tricantiulcero-genic effect of large cardamom (fruits of Amomum subulatum Roxb). J Ethnopharmacol. 2001; 75: 89-94.

PubMed: https://www.ncbi.nlm.nih.gov/pubmed/11297839

20. Zhu JZ. Screening of 15 Chinese herbs for promoting gastrointestinal motility. J Chin Medicinal Materials. 2001; 23: 436.

21. Zhang FY. Clinical value of Amomum villosum in the treatment of functional dyspepsia. J Clin Med. 2014; 7: 124-125.

22. Ding BL, Qi QH. Expression changes of signal transduction pathway of enteric nerves-interstitial cells of cajal in rat with deficiency of spleen-q syndrome and therapeutic effects of Xiang Sha Liu Jun Zi decoction. Chin J Sur Integr Traditional and Western Med. 2013; 19: 397-400.

23. Li XG. Experimental study on pharmacologcal action of bornyl acetate. West Chin J Pharmaceutical. 2001; 25: 49.

24. Wu XS, Li XG, Xiao F. Study on analgesic and anti-inflammatory effects of bornyl acetate in volatile oil of Fructus Amomi. J Chin Medicinal Materials. 2004; 27: 438-439.

25. Zhang T, Lu HS, Yang XX. Volatile oil from Amomi Fructus attenuates 5-fluorouracil-induced intestinal mucositis. Front Pharmacol. 2017; 8: 1-13. PubMed: https://www.ncbi.nlm.nih.gov/pubmed/29170638

26. Ding $P$, Fang $Q$, Zhang DY. Comparative study on the pharmacological activities of Amomun villosum Lour and Amomun villosum Lour. Introduced from Yunnan. J Chin Medicinal Materials. 2004; 39: 29-31.

27. Lee YG, Park JH, Jeon ES. Fructus Amomi Cardamomi Extract Inhibits Coxsackievirus-B3 Induced Myocarditis in a Murine Myocarditis Model. J Microbiol Biotechnol. 2016; 26: 2012-2018.

28. Guo DJ, Cheng HL, Chan SW. Antioxidative activities and the total phenolic contents of tonic Chinese medicinal herbs. Inflammopharmacology. 2008; 16: 201-207.

PubMed: https://www.ncbi.nlm.nih.gov/pubmed/18815744

29. Zhao J, Zhu Y, Dong Z. Antioxidative and antinitrosative effects of volatile oil from AmomumlongiligulareT. L. Wu on ulcerative colitism ice. Chin Traditional Patent Medi. 2009; 31: 1334-1338.

30. Zhang D, Li S, Xiong Q. Extraction characterization and biological activities of polysaccharides from Amomum villosum. Carbohydr Polym. 2013; 5: 114-122.

PubMed: https://www.ncbi.nlm.nih.gov/pubmed/23618247

31. Xiong L, Hu CJ, Shuai XC. Comparative study on the effect of Fructus Amomi on reducing urine before and after salt sunburn. $J$ Chengdu Medical College. 2009; 4: 105-106.

32. Huang FT, Wan MJ, Zhang DY. Chemical composition analysis of the oil in Fructus Amomi leaf and its effect on promoting wound healing. $J$ Guangdong Pharmaceutical University. 2017; 33: 466-470.

33. Zhao RJ, Zhao ZL, Jin MH. Hypoglycemic effect of Fructus Amomi extract on experimental diabetic rats. J Medical Science Yanbian University. 2006; 29: 97-99.

34. Lee SH, Kim JY, Kim H. Amomum villosum induces longitudinal bone 5 in adolescent female rats. J Tradit Chin Med. 2012; 32: 1-2. PubMed: https://www.ncbi.nlm.nih.gov/pubmed/23297572

35. Gao LL, Wang Q, Zhang JW. Study on the purification of flavonoids and their bacteriostatic and antitumor functions in Amomun villosum Lour and Sharpleaf Galangal Fruit. J Food Safety Quality. 2019; 10: 4659-4666. 